Cipango Cahiers d'études japonaises

19 | 2012

Le Japon et le fait colonial II

\title{
Les débats du temps post-colonial, des années 1950 à nos jours
}

Arnaud Nanta et Laurent Nespoulous

\section{OpenEdition}

Journals

Édition électronique

URL : https://journals.openedition.org/cipango/1639

DOI : $10.4000 /$ cipango. 1639

ISSN : 2260-7706

Éditeur

INALCO

\section{Édition imprimée}

Date de publication : 30 octobre 2012

Pagination : 7-13

ISBN : 978-2-85831-204-7

ISSN : $1164-5857$

\section{Référence électronique}

Arnaud Nanta et Laurent Nespoulous, "Les débats du temps post-colonial, des années 1950 à nos jours », Cipango [En ligne], 19 | 2012, mis en ligne le 27 mars 2014, consulté le 30 juin 2021. URL: http://journals.openedition.org/cipango/1639; DOI : https://doi.org/10.4000/cipango.1639

Ce document a été généré automatiquement le 30 juin 2021.

\section{(c) (†) 8}

Cipango est mis à disposition selon les termes de la Licence Creative Commons Attribution - Pas d'Utilisation Commerciale 4.0 International. 


\title{
Les débats du temps post-colonial, des années 1950 à nos jours
}

\author{
Arnaud Nanta et Laurent Nespoulous
}

1 Comme annoncé dans l'éditorial du numéro 18 de Cipango, ce numéro 19 constitue le deuxième volume de notre projet éditorial « Le Japon et le fait colonial ». Il sera cette fois-ci question du temps postcolonial, au sens le plus littéral, et donc chronologique de ce terme. Le lecteur voudra bien se reporter à l'éditorial du précédent numéro afin de prendre connaissance du cadre général, historique et historiographique partagé par ces deux numéros.

2 La date du 15 août 1945 constitue un feuilleté de sens fort complexe. Elle marque, d'une part, la défaite militaire du Japon dans la guerre de l'Asie et du Pacifique (1937-1945), et d'autre part la perte de son empire colonial, tel que décidé lors de la Conférence du Caire (janvier 1942). Pour la Corée, cette fois-ci, le 15 août 1945 constitue la date anniversaire de la chute du joug colonial, désignée par le terme kwangbok 광복 en coréen, c'est-à-dire « le retour de la lumière ». Cette expression, qui est peu employée ailleurs dans le monde sinisé - et pas du tout au Japon -, est également en usage à Taiwan, où elle est lue guangfu 光復 en chinois. Dans ce dernier cas, il n'est pourtant pas question d'« indépendance ", puisque l'île revint dans le giron de la République de Chine (avec laquelle le Japon renoue le dialogue en 1956), pour être à nouveau séparée du continent après 1949. Ce qui ne signifie évidemment pas que la question coloniale ne se poserait pas à Taiwan : elle y constitue, au contraire, un très complexe tissu dans lequel vient en outre se mêler la mémoire de la guerre portée par les Chinois «continentaux » nouvellement arrivés dans l'île. Par ailleurs, les autres anciens territoires coloniaux japonais changent de maîtres: Sakhaline et les Kouriles sont intégrées au territoire russe, la Mandchourie passe sous contrôle chinois, plus précisément de la République populaire après 1949, pour être finalement culturellement sinisée. Enfin, les îles de Micronésie devinrent des États « associés » des États-Unis d'Amérique.

3 Les deux Corées constituent donc, au sein de ces différents territoires, les seuls cas où l'on voit réellement se dérouler un dialogue entre l'ancienne métropole et des États 
souverains composés clairement par les anciens colonisés. La question du dialogue se pose, dans ces cas précis, de façon évidente, par exemple entre la France et l'Algérie ou le Viêtnam. Soulignons de plus que quand «la Corée» est évoquée, il s'agit en réalité presque toujours uniquement de la Corée du Sud (avec qui le Japon a normalisé ses relations diplomatiques en 1965). La Corée du Nord, pourtant tout aussi essentielle quand on réfléchit à l'histoire de la péninsule en général, et à la période coloniale en particulier, est trop souvent oubliée.

4 L'après-décolonisation en Asie de l'Est n'est pas un moment de paix ni d'unité nationale retrouvée. La présence ou l'occupation par le Japon des colonies françaises, britanniques et hollandaises est l'une des causes importantes des guerres coloniales qui suivirent, dans un effort de "repacification » d'espaces en réalité déjà perdus, comme le montra la guerre d'Indochine (1946-1954). Là non plus, les anciennes colonies japonaises ne constituent en rien des contre-exemples. Tout d'abord parce que les indépendances ne furent pas acquises, exception faite de la Corée, pour laquelle l'indépendance avait été promise au Caire en 1942. Ensuite, du fait de la violence qui s'abattit sur toutes ces régions : répression implacable à Taiwan à partir de février 1947 (" incident du 28 février »), puis dans le sud de la Corée, où elle culmine à Cheju (faits connus sous le nom d'« incidents du 3 avril [1948]»); continuation de la guerre civile chinoise et, enfin, fratricide Guerre de Corée (1950-1953), qui aurait fait au moins un million de morts. Si la Seconde Guerre mondiale était "achevée ", l'Asie de l'Est poursuivait, elle, des "guerres l'après-guerre », pour ensuite entrer dans une longue période de division dont on ne voit toujours pas la fin.

5 Ce contexte général, dans lequel le passé colonial s'entremêle avec le contexte de l'après-guerre - c'est-à-dire celui de la guerre froide -, et des conflits "chauds", compliqua lourdement la question du règlement du passé, qui constitue l'un des thèmes importants de ce volume de Cipango. Si le Japon, comme la communauté internationale d'ailleurs, s'accommoda longtemps de régimes dictatoriaux - dits « nationalistes » - en Asie orientale et méridionale (Taiwan, Corée du Sud, voire Viêtnam Sud), au nom de la "guerre contre le communisme" (Chine populaire, Corée du Nord, URSS), la démocratisation à Taiwan, après 1988, et en Corée du Sud, après 1992, ont entraîné un renouveau du débat sur les questions coloniales, lesquelles étaient étouffées dans ces deux pays du temps des régimes militaires. Le personnel politique japonais, notamment les membres importants du Parti libéral-démocrate, du reste, fut longtemps dans le déni quant à la question des responsabilités coloniales (position qui n'a d'ailleurs que peu changé).

6 Venons-en aux contributions composant le présent numéro. La première concerne Taiwan, et les quatre suivantes se déplacent vers la Corée, « perle » de l'empire colonial japonais qui a continué d'occuper, depuis sa scission en deux États ennemis en 1948, une place singulière dans la politique étrangère japonaise, tout comme dans les débats sur la responsabilité coloniale.

7 Dans sa contribution sur le Taiwan postcolonial, qu'il faut aussi saisir comme le Taiwan d'après-guerre, Misawa Mamie 三澤真美恵, historienne sinologue à l'université Nihon, à Tōkyō, revient sur le processus de construction identitaire dans l'île après 1945, entre décolonisation japonaise et intégration dans la République de Chine. En effet, si le Guomindang, qui prit le contrôle de l'île à l'automne 1945, avait combattu le Japon entre 1937 et 1945 (notamment à Shanghai et Nankin), l'expérience des Taiwanais «insulaires» était tout autre, et ceux-ci voyaient 1945 comme une sortie de 
colonisation. L'histoire du cinéma à Taiwan, dont l'auteur est la meilleure spécialiste japonaise, illustre de façon éclairante les relations complexes entre les différentes populations constituant de facto le peuplement de l'île depuis le repli de Chiang Kaishek en 1949. Comment interpréter le succès des films militaires japonais projetés à Taipei en 1960 ? L'opposition entre "insulaires» et "continentaux» offrira une importante clef de compréhension, à moins que ce succès ne soit, à l'inverse, un révélateur des tensions intercommunautaires dans l'île.

8 L'un des points de débat incontournables entre la Corée du Sud, la Corée du Nord et le Japon a trait à la légalité des traités signés entre l'État coréen et l'État japonais entre 1904 et 1910, concernant le protectorat et l'annexion. Arnaud Nanta montre que la polémique n'est pas uniquement portée par les politiques, mais touche bien également les historiens - à la différence de la question des "femmes de réconfort ", laquelle fait consensus parmi les universitaires -, et qu'elle a, en outre, traversé tout le $\mathrm{xx}^{\mathrm{e}}$ siècle. Cette question fut en effet d'abord soulevée en 1919, à Versailles, où les émissaires coréens ne furent toutefois pas autorisés par la France et l'Angleterre à présenter leurs requêtes. Il en fut d'ailleurs de même pour les émissaires algériens. Elle réapparut avec acuité en 1951, lors de la signature du Traité de San Francisco - ou Anglais et Américains repoussèrent les requêtes coréennes -, pour s'effacer à nouveau après le Traité de normalisation diplomatique entre la Corée du Sud et le Japon, en 1965. Son actualité ne s'est pourtant jamais démentie, et elle semble même être un point de cristallisation de la critique coréenne sur le passé colonial japonais.

9 Samuel Guex se penche, quant à lui, sur le débat entre la Corée du Sud et le Japon autour des manuels d'histoire pour le collège. L'auteur part du cas japonais, qu'il a déjà traité par le passé, pour se pencher sur les textes scolaires sud-coréens. Il analyse une série de points historiographiques et la façon dont ceux-ci sont présentés en Corée du Sud, en rappelant le poids qu'y occupe le manuel d'État, malgré la libéralisation de la fin de la décennie 1990. Cette contribution permet, nous semble-t-il, de mieux cerner la complexité de la "guerre des manuels", qui ne peut être réduite à une vulgaire opposition entre pays.

10 Continuant le propos sur la Corée, Lionel Babicz examine l'histoire de la colonisation japonaise et les débats mémoriels autour de celle-ci à la lumière de l'expansion coloniale mondiale qui prit place aux $\mathrm{XIX}^{\mathrm{e}}$ et $\mathrm{xx}^{\mathrm{e}}$ siècles. L'auteur compare terme à terme les couples Japon/Corée et France/Algérie, afin de cerner les différences et surtout les similitudes dans les relations caractérisant ces deux cas d'étude. Si les faits sont évidemment irréductibles à des situations toutes particulières, Lionel Babicz rappelle, dans une analyse dont la portée pédagogique n'est pas à négliger, les limites d'un travail historien insuffisamment mis en perspective avec les grandes dynamiques de l'Époque au sein de laquelle les pays concernés sont inévitablement intégrés.

11 Adrien Carbonnet, enfin, se penche sur le poids qu'a occupé la question coloniale au sein des négociations menées, depuis 1991, entre le Japon et la Corée du Nord. Si la rhétorique nord-coréenne, dans sa grandiloquence, mentionne fréquemment "l'impérialisme nippon », le Japon, membre de l'Alliance américaine, considère, quant à lui, la République populaire démocratique de Corée du Nord comme un "État voyou " ( «rogue state), selon la formule de George W. Bush. Les premières négociations des années 1990 avaient pour objectif de normaliser les relations entre les deux États. Elles ont été interrompues à la fois du fait des événements du 11 septembre et de la question des enlèvements de citoyens japonais par la Corée du Nord, comme l'explique l'auteur. 

par le professeur Kawamura Minato 川村湊, de l'université Hōsei, le 16 décembre 2009 à l'université Toulouse Le Mirail, dans le cadre du colloque « Regards sur les colonies et la colonisation japonaise : histoires et représentations ", ainsi que lors de l'assemblée générale de la Société française des études japonaises le 19 décembre 2009 à l'Inalco. Il y évoque les traces et la mémoire de la colonisation dans la littérature japonaise du $\mathrm{Xx}^{\mathrm{e}}$ siècle.

Quel regard général porter sur ces deux numéros de Cipango consacrés au fait colonial japonais? Nous le soulignions dans notre éditorial du numéro 18 : il est impossible de restituer une sorte de grande mécanique générale qui caractériserait tout ce qui est origine et conséquence de la colonisation japonaise. Cette remarque est d'ailleurs valable pour les autres colonisations. Du dernier tiers du XIx ${ }^{\mathrm{e}}$ siècle jusqu'à la moitié du $\mathrm{xx}^{\mathrm{e}}$ siècle, tout d'abord, des facteurs contradictoires ont joué autant qu'une planification bien posée, laquelle planification peut, du reste, n'être qu'une illusion provoquée sciemment, a posteriori, par les autorités coloniales, afin de donner du sens à leur action. La colonisation n'est pas un bloc monolithique, mais un phénomène dynamique et protéiforme, qui se développe tantôt en adéquation avec son terrain d'élection (ce que démontrent les politiques, au pluriel, de développement), tantôt contre ses propres intérêts à moyen ou long terme (ce que virent, fort tôt, certains critiques et commentateurs japonais). Le temps postcolonial est, bien logiquement, à cette image lui aussi : à des colonisations différentes, correspondent des destins postcoloniaux variés, allant de cas de changement de «tutelle " à des situations de création de véritables États souverains. Un autre sujet, qui illustre bien ces disparités de la « reformulation » des anciens territoires colonisés, abordé ici surtout au sujet du Taiwan d'après 1945, est celui de la constitution des ethnicités modernes. Tous les anciens colonisés formulent une nouvelle identité, qui n'a plus guère à voir avec ce qu'elle était auparavant, et qui est le fruit tourmenté d'une histoire coloniale, d'une histoire de guerre et souvent encore, d'une histoire de conflits fratricides.

14 L'Asie orientale présente ainsi une configuration singulière: là où les anciennes puissances coloniales européennes se côtoient ou se font la guerre sur un pied d'égalité, si l'on peut dire, le Japon est la seule puissance coloniale, au sens moderne, de sa région, et est, par conséquent, entouré exclusivement d'États modernes avec lesquels il a entretenu des relations inégales, que ce soit dans la guerre ou au travers de la domination coloniale. Cela complique la nature du dialogue.

Notre objet était de permettre, dans une certaine mesure, d'illustrer la complexité, parfois aussi les ambiguïtés, de la situation et des discours tant du colonisateur que du colonisé, tant de l'ancien colonisateur que de l'ancien colonisé. 